Copyright (C) 2009. IEEE. Personal use of this material is permitted. Permission from IEEE must be obtained for all other uses, in any current or future media, including reprinting/republishing this material for advertising or promotional purposes, creating new collective works, for resale or redistribution to servers or lists, or reuse of any copyrighted component of this work in other works. 


\title{
Data Association and Track Management for the Gaussian Mixture Probability Hypothesis Density
} Filter

\author{
Kusha Panta, Student Member, IEEE, Daniel E. Clark and Ba-Ngu Vo
}

\begin{abstract}
The Gaussian Mixture Probability Hypothesis Density (GM-PHD) recursion is a closed-form solution to the probability hypothesis density (PHD) recursion, which was proposed for jointly estimating the time-varying number of targets and their states from a sequence of noisy measurement sets in the presence of data association uncertainty, clutter and miss-detection. However the GM-PHD filter does not provide identities of individual target state estimates, that are needed to construct tracks of individual targets. In this paper, we propose a new multi-target tracker based on the GM-PHD filter, which gives the association amongst state estimates of targets over time and provides track labels. Various issues regarding initiating, propagating and terminating tracks are discussed. Furthermore, we also propose a technique for resolving identities of targets in close proximity, which the PHD filter is unable to do on its own.
\end{abstract}

\section{Index Terms}

Multi-target Tracking, Random Sets, Probability Hypothesis Density (PHD) Filter, Gaussian Mixture.

\section{INTRODUCTION}

Multi-target tracking (MTT) involves the joint estimation of an unknown and time-varying number of targets as well as their individual states from a sequence of sets of noisy measurements with uncertain data association [1], [2]. The number of targets changes over time as new targets appear (i.e., target birth process) or existing targets disappear (i.e., target death). The number of measurements also varies over time as not all existing targets generate measurements and a number of measurements may be clutter, i.e., spurious measurements that are not generated by any of the existing targets. Assuming targets move independently of one another, tracking them with separate

Manuscript received Month xx, 200x; revised Month xx, 200x.

Kusha Panta is with Co-operative Research Centre for Sensor and Information Processing (CSSIP), Department of Electrical and Electronic Engineering, The University of Melbourne, Melbourne, Victoria 3010, Australia. Email: k.panta@ee.unimelb.edu.au.

Daniel E. Clark is with Department of Electrical Engineering, Heriot-Watt University, Riccarton, Edinburgh, United Kingdom. Email: dec30@cantab.net.

Ba-Ngu Vo is with Department of Electrical and Electronic Engineering, The University of Melbourne, Melbourne, Victoria 3010, Australia. Email: b.vo@ee.unimelb.edu.au. 
filters requires correct association of measurements with individual targets over time [1], [2], [3]. The problem of correctly associating measurements to targets over time is a data-association problem and requires various ad-hoc methods in practice to stop the associated cost from growing exponentially over time.

Random Finite Set (RFS) theory offers a mathematically elegant and natural representation of finite, timevarying number of targets and measurements [6], [7], [8], [9]. Using RFSs to model the collections of targets and measurements, Mahler's finite set statistics (FISST) provides a rigorous Bayesian framework for multi-target tracking. This has lead to the development of a number of novel multi-target filters, such as the multi-target Bayes filter [6], [9], the sequential Monte Carlo (SMC) multi-target Bayes filter [11], [12], [15], [16], the probability hypothesis density (PHD) filter [9], and the SMC-PHD filter (also known as the particle-PHD filter) [11], [12], [17], [18].

The optimal multi-target Bayes filter based on RFS theory is, in general, computationally intractable for it requires evaluating combinatorial sums of integrals of high dimensions with a prohibitively large number of combinations even for medium number of targets. The PHD filter is a suboptimal but computationally tractable alternative to the RFS Bayes multi-target filter [9]. It is a recursion that only propagates the PHD or the intensity function of the RFS of targets. The PHD recursion operates on the single-target state space and avoids the explicit problem of data association. Though the PHD recursion consists of equations that are considerably simpler than those of the optimal multi-target Bayes filter, it still requires solving multi-dimensional integrals that do not have closed-form solutions in general. A generalized SMC implementation of the PHD filter (SMC- or particle-PHD filter) has been proposed in [11], [12]. Similar SMC implementations of the PHD filter have also been proposed in [17], [18]. Data association for the SMC-PHD filter has been considered in [19], [20], [21], [22], [23].

Recently, a closed-form solution to the PHD recursion, called the Gaussian-Mixture Probability Hypothesis Density (GM-PHD) filter, has been derived for jointly estimating the time-varying number of targets and their states recursively from the sequence of noisy measurements sets in the presence of clutter and association uncertainty for linear Gaussian multi-target models [13], [14]. The posterior PHD function is approximated by a sum of weighted Gaussian components whose weights, means and covariances are propagated analytically in time. Interestingly, the mean and covariance of each Gaussian component are propagated by the Kalman filter. The GM-PHD filter has been extended to accommodate non-linear target dynamical models, thereby providing a computationally efficient solution for multi-target filtering problems. However, the GM-PHD filter, like the SMC-PHD filter, only provides identity-free estimates of target states and hence no temporal association of estimates over time.

This paper addresses the issue of temporal association for state estimates of individual targets. We propose a GM-PHD filter based multi-target tracker that provides not only the state estimates of targets at each time step but also association of state estimates to targets over time so that estimates of state trajectories of individual targets can be obtained. It is shown here that state trajectories of the individual targets can be determined directly from the evolution of the Gaussian mixture and that single Gaussians within the mixture accurately track individual targets. The methods proposed here are implemented separately without affecting the GM-PHD recursion. For illustration 
purposes, we demonstrate the ability of the proposed tracker to estimate the correct number of targets and their trajectories in high density clutter. The performance of the GM-PHD filter is benchmarked against the multiple hypothesis tracker (MHT) and is shown to outperform the MHT in terms of its ability to pick up the correct number tracks and their trajectory estimates. Finally, we also consider the issue of resolving identities of targets in proximity for the PHD filter and propose a technique for doing so.

The rest of the paper is organized as follows. Section II provides a summary of multi-target models and the PHD filter. Section II also presents the Gaussian linear multi-target models for which the GM-PHD recursion holds and a summary of the GM-PHD filter. Section III presents the GM-PHD tracker proposed in this paper. For illustration purposes, simulation results for the GM-PHD tracker have been presented in Section V. Section V also discusses the performance of the GM-PHD tracker, as benchmarked against the MHT. Section VI proposes a new technique for resolving the identities of targets in proximity using the GM-PHD filter. Finally, Section VII presents concluding remarks and outlines future research directions.

\section{BACKGROUND}

This section describes describes a generic multi-target tracking/filtering problem which applies to wide range of multi-target tracking problems.

\section{A. Multi-Target Model}

In a multi-target tracking scenario, targets appear and disappear randomly. New targets appear in the surveillance region either due to spontaneous target birth or targets spawned by existing targets. The number of targets born at each instant is assumed to follow a Poisson distribution with a mean of $\lambda_{b}$. A target present at time step $k$ may not survive to the next time step. Target death is modelled with a probability of $1-p_{S, k}\left(x_{k-1}\right)$, where $p_{S, k}\left(x_{k-1}\right)$ represents the probability that a target of state $x_{k-1}$ at time step $(k-1)$ will survive to the next time step. For the duration the target is present in the surveillance region, it moves according to a Markov dynamic model

$$
x_{k} \sim f_{k \mid k-1}\left(\cdot \mid x_{k-1}\right)
$$

and if detected, generates at most one observation according to

$$
z_{k} \sim g_{k}\left(\cdot \mid x_{k}\right)
$$

The probability that a target of state $x_{k}$ being detected is $p_{D, k}\left(x_{k}\right)$.

At time step $k$, let $N_{k}$ be the number of targets with states, $x_{k, 1}, \ldots, x_{k, N_{k}}$, and $M_{k}$ the number of observations received, $z_{k, 1}, \ldots, z_{k, M_{k}}$. Then $x_{k, i}$ denotes the state of $i^{t h}$ target and $z_{k, j}$ denotes $j^{\text {th }}$ observation received. Let

$$
\begin{aligned}
& X_{k}=\left\{x_{k, 1}, \ldots, x_{k, N_{k}}\right\} \subset \mathcal{X}, \\
& Z_{k}=\left\{z_{k, 1}, \ldots, z_{k, M_{k}}\right\} \subset \mathcal{Z},
\end{aligned}
$$


denote the collection of targets and observations received at time step $k$, where $\mathcal{X} \subseteq \mathbb{R}^{n_{x}}$ and $\mathcal{Z} \subseteq \mathbb{R}^{n_{z}}$ represent the state and observation space where individual targets and observations, respectively, lie. Some of the $M_{k}$ observations may be due to clutter. If $z_{k, i}$ is due to clutter, it follows a clutter probability density $c_{k}(\cdot)$. The number of clutter points are assumed to be Poisson distributed with a mean of $\lambda_{c}$. Further assumptions required for the GM-PHD filter will be given in Section II-C.

\section{B. Random Finite Set (RFS) and the Probability Hypothesis Density (PHD) Filter}

In the RFS framework, collections of targets $X_{k}$ and observations $Z_{k}$ are treated as a multi-target state and multi-target observation. Uncertainty in a multi-target system is then characterized by modelling $X_{k}$ and $Z_{k}$ as random finite sets. A RFS $X$ is a finite set-valued random variable, i.e., individual elements as well as the number of elements are random. The probability law of the RFS $X$ can be specified by a discrete distribution and a family of joint distributions [4]. The discrete distribution characterizes the number of elements of $X$ (i.e. $|X|$ ) while each of the joint distributions characterizes the elements in $X$ given its cardinality. Readers should refer to [8], [9], [12], [14] for details on the formulation of the RFS framework for multi-target state estimation.

For a RFS $X$ on $\mathcal{X}$ with a probability distribution $\mathcal{P}$, its first order moment, is a non-negative function $v$ on $\mathcal{X}$, called the intensity or the PHD function, with the property that for any closed subset $S \subseteq \mathcal{X}$ [4], [5]

$$
\int_{S} v(x) d x=\int|X \cap S| \mathcal{P}(d X)
$$

where $|X|$ denotes the cardinality of $X$. Given the intensity function $v$, its integral over any region $S$ gives an estimate for the number of elements in $X$ that are present in $S$. The local maxima of the intensity function $v$ are points in $\mathcal{X}$ with the highest local concentration of expected number of elements, and hence can be used to generate estimates for the elements of $X$.

The PHD filter is a less computationally expensive alternative to propagating the multi-target posterior density recursively in time. It propagates the posterior intensity function of the multi-target RFS as follows [9]: given the posterior intensity $v_{k-1}$ at time step $k-1$, the intensity function $v_{k \mid k-1}$ to time step $k$ is given as

$$
\begin{aligned}
& v_{k \mid k-1}(x)=\int\left[p_{S, k}(\xi) f_{k \mid k-1}(x \mid \xi)+\right. \\
& \left.\beta_{k \mid k-1}(x \mid \xi)\right] v_{k-1}(\xi) d \xi+\gamma_{k}(x),
\end{aligned}
$$

and the posterior intensity $v_{k}$ at time step $k$ is given as

$$
\begin{aligned}
v_{k}(x) & =\left[1-p_{D, k}(x)\right] v_{k \mid k-1}(x) \\
& +\sum_{z \in Z_{k}} \frac{p_{D, k}(x) g_{k}(z \mid x) v_{k \mid k-1}(x)}{\kappa_{k}(z)+\int p_{D, k}(\xi) g_{k}(z \mid \xi) v_{k \mid k-1}(\xi) d \xi},
\end{aligned}
$$

where $\kappa_{k}(\cdot)$ is the intensity of the clutter RFS and equals $\lambda_{c} c_{k}(\cdot) ; Z_{k}$ is the multi-target observation available at time step $k ; \gamma_{k}(\cdot)$ denotes the intensity of spontaneous target birth; $\beta_{k \mid k-1}(\cdot \mid \xi)$ denotes the intensity of the target RFS spawned by a target of previous state $\xi$ at time step $k$; and definitions of $p_{S, k}(\cdot), f_{k \mid k-1}(\cdot \mid \cdot), g_{k}(\cdot \mid \cdot), c_{k}(\cdot), \lambda_{c}$ and $p_{D, k}(\cdot)$ are the same as the ones given in Section II-A. 
For the recursion given in (5)-(6), the following assumptions hold:

1) Each target evolves and generates an observation independently of one another.

2) The clutter RFS is Poisson (or distribution on the number of clutter is Poisson) and is independent of targetoriginated observations. A RFS $X$ is a Poisson process with the mean $N=\int v(x) d x$. Given $v(x)$, elements of $X$ are i.i.d. according to $v / N$. Thus a Poisson RFS is completely characterized by its intensity function.

3) The predicted multi-target RFS is Poisson.

First two assumptions are common in most multi-target applications [1], [3]. The third assumption is a simplification needed to derive the PHD update and is a reasonable one in tracking scenarios where interactions amongst targets are negligible.

The PHD recursion given in (5)-(6) does not admit analytical solutions in general and can be approximated by some numerical integration methods such as sequential Monte Carlo (SMC) [11], [12], [17]. However, a closed-form solution, called the Gaussian Mixture PHD (GM-PHD) filter has recently been presented in [13], [14] for linear Gaussian multi-target models. It is summarized next.

\section{Linear Gaussian Multi-Target Models}

The linear Gaussian multi-target models for which a closed-form solution to the PHD recursion exists are given as follows:

1) Each target follows a linear Gaussian model, i.e.,

$$
\begin{aligned}
f_{k \mid k-1}(x \mid \zeta) & =\mathcal{N}\left(x ; F_{k-1} \zeta, Q_{k-1}\right) \\
g_{k}(z \mid x) & =\mathcal{N}\left(z ; H_{k} x, R_{k}\right)
\end{aligned}
$$

where $\mathcal{N}(\cdot ; m, P)$ denotes the Gaussian density with mean $m$ and covariance $P, F_{k-1}$ is the state transition matrix and $Q_{k-1}$ is the process noise covariance, $H_{k}$ is the observation matrix, and $R_{k}$ is the observation noise covariance.

2) The survival and detection probabilities are both state independent, i.e.,

$$
\begin{gathered}
p_{S, k}(x)=p_{S, k}, \\
p_{D, k}(x)=p_{D, k} .
\end{gathered}
$$

3) The intensities of the birth and spawn RFSs are both Gaussian mixtures of the form

$$
\begin{gathered}
\gamma_{k}(x)=\sum_{i=1}^{J_{\gamma, k}} w_{\gamma, k}^{i} \mathcal{N}\left(x ; m_{\gamma, k}^{i}, P_{\gamma, k}^{i}\right), \\
\beta_{k \mid k-1}(x \mid \zeta)=\sum_{j=1}^{J_{\beta, k}} w_{\beta, k}^{j} \mathcal{N}\left(x ; F_{\beta, k-1}^{j} \zeta+d_{\beta, k-1}^{j}, Q_{\beta, k-1}^{j}\right)
\end{gathered}
$$

where $J_{\gamma, k}, w_{\gamma, k}^{i}, P_{\gamma, k}^{i}, P_{\gamma, k}^{i}, i=1, \ldots, J_{\gamma, k}$ are given model parameters that determine the shape of the birth intensity; similarly $J_{\beta, k}, w_{\beta, k}^{j}, F_{\beta, k-1}^{j}, d_{\beta, k-1}^{j}, Q_{\beta, k}^{j}, j=1, \ldots, J_{\beta, k}$ determine the shape of the spawning 
intensity of a target with the previous state $\zeta$. Readers should refer to [14] for further remarks on these assumptions.

\section{The Gaussian Mixture PHD (GM-PHD) Filter}

For linear Gaussian multi-target models given in Section II-C, the PHD recursion of (5)-(6) yields a closed-form solution called the GM-PHD recursion which consists of the following prediction and update steps.

Prediction Step: Given that the posterior intensity $v_{k-1}$ at time step $k-1$ is a Gaussian mixture of the form

$$
v_{k-1}(x)=\sum_{i=1}^{J_{k-1}} w_{k-1}^{i} \mathcal{N}\left(x ; m_{k-1}^{i}, P_{k-1}^{i}\right),
$$

the predicted intensity to time step $k$ is also a Gaussian mixture and is given by

$$
v_{k \mid k-1}(x)=v_{S, k \mid k-1}(x)+v_{\beta, k \mid k-1}(x)+\gamma_{k}(x),
$$

where $\gamma_{k}(x)$ is given in (11),

$$
\begin{gathered}
v_{S, k \mid k-1}=p_{S, k} \sum_{j=1}^{J_{k-1}} w_{k-1}^{j} \mathcal{N}\left(x ; m_{S, k \mid k-1}^{j}, P_{S, k \mid k-1}^{j}\right), \\
m_{S, k \mid k-1}^{j}=F_{k-1} m_{k-1}^{j}, \\
P_{S, k \mid k-1}^{j}=Q_{k-1}+F_{k-1} P_{k-1}^{j}\left(F_{k-1}\right)^{T}, \\
v_{\beta, k \mid k-1}(x)=\sum_{j=1}^{J_{k-1}} \sum_{l=1}^{J_{\beta, k}} w_{k-1}^{j} w_{\beta, k}^{l} \mathcal{N}\left(x ; m_{\beta, k \mid k-1}^{j, l}, P_{\beta, k \mid k-1}^{j, l}\right), \\
m_{\beta, k \mid k-1}^{j, l}=F_{k-1}^{l} m_{k-1}^{j}+d_{\beta, k-1}^{l}, \\
P_{\beta, k \mid k-1}^{j, l}=Q_{\beta, k-1}^{l}+F_{\beta, k-1}^{l} P_{\beta, k-1}^{j}\left(F_{\beta, k-1}^{l}\right)^{T}
\end{gathered}
$$

and $[\cdot]^{T}$ denotes the transpose of the matrix $[\cdot]$.

Update Step: Assuming that the predicted intensity $v_{k \mid k-1}$ to time step $k$ is a Gaussian mixture of the form

$$
v_{k \mid k-1}(x)=\sum_{i=1}^{J_{k \mid k-1}} w_{k \mid k-1}^{i} \mathcal{N}\left(x ; m_{k \mid k-1}^{i}, P_{k \mid k-1}^{i}\right)
$$

the posterior intensity $v_{k}$ at time step $k$ is also a Gaussian mixture, and is given by

$$
v_{k}(x)=\left(1-p_{D, k}\right) v_{k \mid k-1}(x)+\sum_{z \in Z_{k}} v_{D, k}(x ; z)
$$


where

$$
\begin{gathered}
v_{D, k}(x ; z)=\sum_{j=1}^{J_{k \mid k-1}} w_{k}^{j}(z) \mathcal{N}\left(x ; m_{k \mid k}^{j}(z), P_{k \mid k}^{j}\right), \\
w_{k}^{j}(z)=\frac{p_{D, k} w_{k \mid k-1}^{j} q_{k}^{j}(z)}{\kappa_{k}(z)+p_{D, k} \sum_{l=1}^{J_{k \mid k-1}} w_{k \mid k-1}^{l} q_{k}^{l}(z)}, \\
q_{k}^{j}=\mathcal{N}\left(z ; H_{k} m_{k \mid k-1}^{j}, R_{k}+H_{k} P_{k \mid k-1}^{j}\left(H_{k}\right)^{T}\right), \\
m_{k \mid k}^{j}(z)=m_{k \mid k-1}^{j}+K_{k}^{j}\left(z-H_{k} m_{k \mid k-1}^{j}\right), \\
P_{k \mid k}^{j}=\left[I-K_{k}^{j} H_{k}\right] P_{k \mid k-1}^{j}, \\
K_{k}^{j}=P_{k \mid k-1}^{j}\left(H_{k}\right)^{T}\left(H_{k} P_{k \mid k-1}^{j}\left(H_{k}\right)^{T}+R_{k}\right)^{-1} .
\end{gathered}
$$

The expected number of targets $\widehat{N}_{k \mid k-1}$ and $\widehat{N}_{k}$ associated with $v_{k \mid k-1}$ and $v_{k}$ are obtained by summing the appropriate mixture weights. The closed-form recursions for $\widehat{N}_{k \mid k-1}$ and $\widehat{N}_{k}$ are as follows:

$$
\begin{gathered}
\widehat{N}_{k \mid k-1}=\widehat{N}_{k-1}\left(p_{S, k}+\sum_{j=1}^{J_{\beta, k}} w_{\beta, k}^{j}\right)+\sum_{j=1}^{J_{\gamma, k}} w_{\gamma, k}^{j}, \\
\widehat{N}_{k}=\widehat{N}_{k \mid k-1}\left(1-p_{D, k}\right)+\sum_{z \in Z_{k}} \sum_{j=1}^{J_{k \mid k-1}} w_{k}^{j}(z) .
\end{gathered}
$$

The number of Gaussian terms $J_{k}$ in $v_{k}$ at time step $k$ equals $\left(J_{k-1}\left(1+J_{\beta, k}\right)+J_{\gamma, k}\right)\left(1+\left|Z_{k}\right|\right)$ and increases with time. The pruning and merging techniques proposed in [13], [14] can be used to stop $J_{k}$ from growing with time. Additional schemes for pruning least likely Gaussians terms are given in Section III. The number of Gaussian terms is reduced by either eliminating the Gaussian terms with low weights or by keeping only a certain number of terms with strongest weights. The closely spaced Gaussian terms are also merged into one as they are more efficiently approximated by a single Gaussian term. It has been shown that the errors introduced in the Gaussian mixture reduction techniques can be bounded, ensuring a reasonable approximation [25].

1) Multi-target State Estimation: Given the posterior intensity function at time step $k$ as the mixture of weighted Gaussians with respective means and variances, state estimates of individual targets may be extracted by picking the means of the Gaussian terms with weights greater than an appropriately chosen threshold, $w_{T h}$,

$$
\widehat{X}_{k}=\left\{m_{k}^{i}: w_{k}^{i}>w_{T h}, i=1, \cdots, J_{k}\right\}
$$

As a result, the GM-PHD filter avoids the need for standard clustering techniques that are needed for the SMC-PHD filter. Standard clustering techniques are computationally demanding and their performances suffer when $\widehat{N}_{k}$ differs from the natural number of clusters that is present in the particle approximation of $v_{k}$.

It should be noted that the GM-PHD filter has also been extended to handle nonlinear target dynamical and nonlinear measurement models by replacing the Kalman filter ${ }^{1}$ with its extended and unscented counterparts to

\footnotetext{
${ }^{1}$ The Kalman filter consists of the time prediction step given by (15)-(16) and the measurement update step given by (24)-(26).
} 
propagate means and covariances of Gaussian components [14]. Furthermore, the GM-PHD recursion can also handle state dependent target detection and target survival probabilities [14].

The GM-PHD filter proposed in [13], [14] does not provide the temporal association of estimates, which are needed if estimates of individual target trajectories are required. The following section will show how the GM-PHD filter can be extended into a robust and computationally efficient multi-target tracker that not only provides the state estimates of individual targets but also their identities, which can be used to obtain estimates of target trajectories. Preliminary results of the GM-PHD based tracker first appeared in [24], [26].

\section{The Gaussian Mixture Probability Hypothesis Density (GM-PHD) Tracker}

This section presents a multi-target tracker based on the GM-PHD filter, referred to as the GM-PHD tracker from here onwards. The idea behind the GM-PHD tracker is to assign identities to individual Gaussian terms of the mixture representing the posterior intensity function and to allow these identities to evolve through time without affecting the GM-PHD recursion. This formulation of the GM-PHD tracker was motivated by the formulation of the improved particle PHD filter proposed in [22], which uses additional indices associated with samples to obtain target identities during the clustering of particles.

\section{A. The GM-PHD tracker}

The algorithm for the GM-PHD tracker consists of the following steps:

\section{Step 0: Initialization}

At time step $k=0$, the intensity function, $v_{0}$ is the mixtures of $J_{0}$ Gaussians as

$$
v_{0}(x)=\sum_{i=1}^{J_{0}} w_{0}^{i} \mathcal{N}\left(x ; m_{0}^{i}, P_{0}^{i}\right),
$$

where all of these Gaussians are distributed across the state space. A unique identifier (or tag) is assigned to each Gaussian to form the set

$$
\mathcal{T}_{0}=\left\{\tau_{0}^{1}, \cdots, \tau_{0}^{J_{0}}\right\}
$$

where $\tau_{0}^{j}$ denotes the tag of the $j^{\text {th }}$ Gaussian term with mean, $m_{0}^{j}$ and covariance $P_{0}^{j}$.

\section{Step 1: Prediction}

Predict the intensity forward in time according to (13) and construct the set of new tags as follows:

$$
\mathcal{T}_{k \mid k-1}=\mathcal{T}_{k-1} \cup\left\{\tau_{\gamma, k}^{1}, \cdots, \tau_{\gamma, k}^{J_{\gamma, k}}\right\} \cup\left\{\tau_{\beta, k}^{1,1}, \cdots, \tau_{\beta, k}^{J_{k-1}, J_{\beta, k}}\right\}
$$

where $m_{k \mid k-1}^{i}$ retains the tag of its prior $m_{k-1}^{i}, \tau_{\gamma, k}^{i}$ is the new tag associated with $i^{\text {th }}$ Gaussian term introduced by birth process and $\tau_{\beta, k}^{i, j}$ is the tag of $j^{\text {th }}$ Gaussian term spawned by $i^{\text {th }}$ Gaussian term of the mixture. Here, a new tag is given to each new spawned Gaussian term.

\section{Step 2: Update}

Update the predicted intensity according to (20). 
Each term in the predicted Gaussian mixture gives rise to $\left(1+\left|Z_{k}\right|\right)$ terms in the updated mixture and we assign the same tag to each of the updated Gaussian terms as its associated predicted term, i.e., $m_{k}^{j}(z)$ gets the same tag as that of $m_{k \mid k-1}^{j}$ for each $z$ or no observation. As a result, we have a multiple number of updated Gaussian terms for every predicted Gaussian term and their weights (see Figure 1).

\section{Step 3: Pruning and Merging}

Pruning of Gaussian terms is performed by either removing the Gaussian terms with low weights or keeping a certain number of terms with strongest weights. The Gaussian terms that are within a certain distance of each other are also merged into one.

Given a truncation threshold $T_{T h}$, a merging threshold $U$ and a maximum allowable number number of Gaussian terms $J_{\max }$, the number of terms in the Gaussian mixture at each time step is pruned as follow.

Set $l=0$, and $I=\left\{i: 1 \leq i \leq J_{k}, w_{k}^{i}>T_{T h}\right\}$,

Repeat

$$
\begin{aligned}
& l:=l+1 \\
& j:=\arg \max _{i \in I} w_{k}^{i} \\
& L:=\left\{i \in I \mid\left(m_{k}^{i}-m_{k}^{j}\right)^{T}\left(P_{k}^{i}\right)^{-1}\left(m_{k}^{i}-m_{k}^{j}\right) \leq U\right\} \\
& \tilde{w}_{k}^{l}=\sum_{i \in L} w_{k}^{i} \\
& \tilde{m}_{k}^{l}=\frac{1}{\tilde{w}_{k}^{l}} \sum_{i \in L} w_{k}^{i} m_{k}^{i} \\
& \tilde{P}_{k}^{l}=\frac{1}{\tilde{w}_{k}^{l}} \sum_{i \in L} w_{k}^{i}\left(P_{k}^{i}+\left(\tilde{m}_{k}^{l}-m_{k}^{i}\right)\left(\tilde{m}_{k}^{l}-m_{k}^{i}\right)^{T}\right) \\
& I:=I \backslash L
\end{aligned}
$$

Until $I=\emptyset$.

If $l>J_{\text {max }}$, replace $\left\{\tilde{w}_{k}^{i}, \tilde{m}_{k}^{i}, \tilde{P}_{k}^{i}\right\}_{i=1}^{l}$ by those of the $J_{\text {max }}$ Gaussian terms with largest weights and output $\left\{\tilde{w}_{k}^{i}, \tilde{m}_{k}^{i}, \tilde{P}_{k}^{i}\right\}_{i=1}^{\tilde{J}_{k}}$ with $\tilde{J}_{k}=\min \left(J_{\max }, J_{k}\right)$.

where $\left\{\tilde{w}_{k}^{i}, \tilde{m}_{k}^{i}, \tilde{P}_{k}^{i}\right\}_{i=1}^{\tilde{J}_{k}}$ denote the remaining Gaussian components after merging. The intensity function after pruning is given by

$$
\tilde{v}_{k}(x)=\sum_{i=1}^{\tilde{J}_{k}} \tilde{w}_{k}^{i} \mathcal{N}\left(x ; \tilde{m}_{k}^{i}, \tilde{P}_{k}^{i}\right)
$$

\section{Step 4: Target State Estimation}

At time step $k$, state estimates of individual target states are given by (29). The set of tags associated with target states is given by

$$
\widehat{T}_{k}=\left\{\tau_{k}^{i}: w_{k}^{i}>w_{T h}\right\} .
$$

A complete and systematic method for the construction and the management of tracks using these tagged Gaussians is given in the following section. 


\section{Tag and Track Management Scheme for the GM-PHD Tracker}

This section presents a scheme for initiating, propagating and terminating tracks for the GM-PHD tracker. For the rest of the paper, a target trajectory means the path in target space followed by a target.

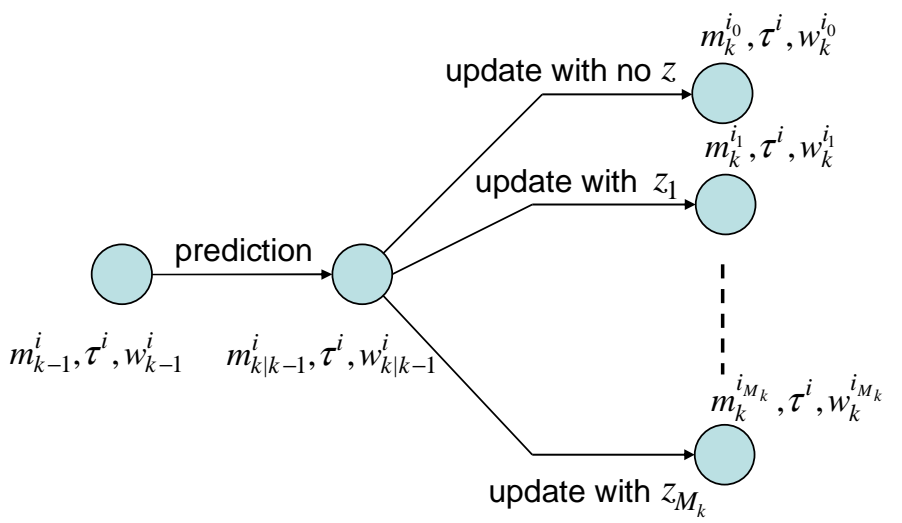

Fig. 1. A part of a tree structure for propagating a Gaussian term and its tag from the previous time step $k-1$ to the time step $k$ given $Z_{k}$.

\section{A. Tag Management Scheme}

As mentioned in Section III, new tags are assigned either for Gaussian terms during initialization or for new Gaussian terms contributed by the birth term $\gamma_{k}$. The propagation of tags associated with individual terms in the Gaussian mixture approximating $v_{k \mid k-1}$ is summarized in Figure 1. (Here $m_{k}^{i_{l}}$ denotes the mean of the Gaussian that results when $m_{k \mid k-1}^{i}$ is updated with measurement $z_{l}$ where $l=0$ represents the case of no measurement update to account for the case of miss-detection). All of the $\left(1+\left|Z_{k}\right|\right)$ updated Gaussians are assigned the same tag as that of its prior, $\tau^{i}$. Over time, each Gaussian initialized at time step $k=0$ and contributed by $\gamma_{k}$ form the root of a tree whose number of branches grows linearly with the number of measurements available at each time step (see Figure 2). Each tree is identified by its unique label that is the same as the tag of the Gaussian term at its root. Each branch of a tree is a possible state trajectory of a target. At the end of the GM-PHD recursion at each time step, we have a number of tree structures where each tree provides a collection of possible tracks of a target. The likelihood of each track is given by its weight. One solution is to pick the branch with the largest weight $w_{k}^{i_{l}}>w_{T h}$ from every tree to form a collection individual target tracks and their respective track labels. For each selected track, its label is the same as that of the tree it belongs to.

For the purpose of devising an efficient scheme for track initiation, propagation and termination, we first classify tree structures as confirmed and tentative ones. A confirmed tree structure is one that has at least one branch with its weight $w_{k}^{j}>w_{T h}$. Otherwise the tree is classified as tentative. All confirmed trees contribute a track and its label towards a collection of output tracks called the track set, $\Im_{k}$. Each member of $\Im_{k}$ is an estimate of a target trajectory with its unique label, i.e., $\left(\left\{m_{k_{i}}^{i}, \cdots, m_{k}^{i}\right\}, \tau^{i}\right)$ where $k_{i}$ denotes the time step at which $i^{\text {th }}$ target enters the surveillance region and $\left\{m_{k_{i}}^{i}, \cdots, m_{k}^{i}\right\}$ represents the trajectory estimate of $i^{\text {th }}$ target. 


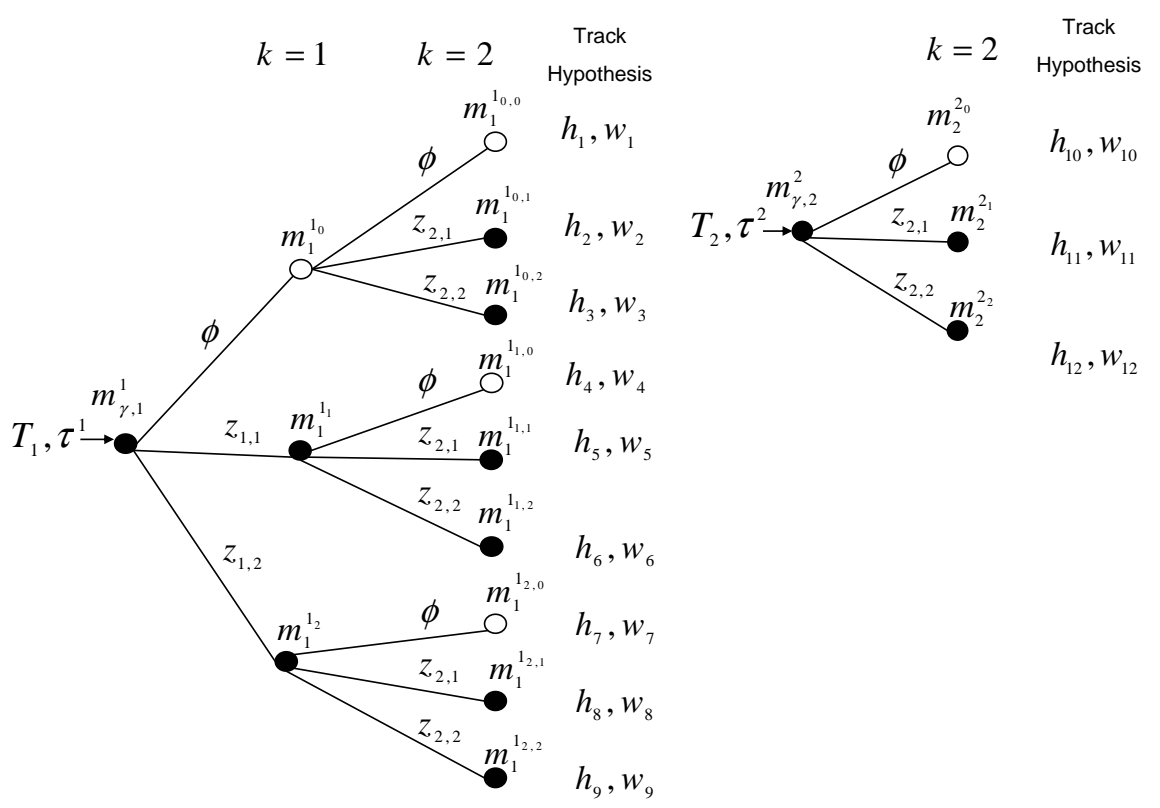

Fig. 2. An example of track-oriented implementation of the GM-PHD Filter. $Z_{1}=\left\{z_{1,1}, z_{1,2}\right\}$ and $Z_{2}=\left\{z_{2,1}, z_{2,2}\right\}, v_{0}=0$ and a Gaussian term each is contributed by $\gamma_{k}$ at both time steps $k=1$ and $k=2$. For simplicity, we denote $w_{k}^{i}$ by $w_{i}$.

For illustration purposes, Figure 2 represents a tracking example where there are no Gaussian terms at initialization. The birth process contributes a Gaussian term each with the mean $m_{\gamma, 1}^{1}$ at time step $k=1$ and $m_{\gamma, 2}^{2}$ at time step $k=2$. A tree with the label $\tau^{1}$ is initialized for $m_{\gamma, 1}^{1}$ at time step $k=1$ and similarly the label $\tau^{2}$ is initialized for $m_{\gamma, 2}^{2}$ at time step $k=2$. At both time steps $k=1$ and $k=2$, there are two measurements each. The branch with $\phi$ denotes the case of miss-detection. At time step $k=2$, tree $T_{2}$ is a tentative track and tree $T_{1}$ is a confirmed track assuming only the branch represented by branch $h_{6}$ has its weight $w_{6}>w_{T h}$.

\section{B. Track Initiation, Propagation and Termination}

1) Track Initiation: At time step $k=0$, we initialize a tree with $m_{0}^{i}$ as its root and $\tau_{0}^{i}$ as its label for $i=1, \cdots, J_{0}$. We also initialize a tree for every Gaussian term $m_{\gamma, k}^{i}$ contributed by $\gamma_{k}$ at time step $k>0$, with its mean $m_{\gamma, k}^{j}$ as its root and is given a label $\tau_{\gamma, k}^{j}$. All trees during initialization are classified as tentative trees.

2) Track Confirmation, Propagation and Termination: At each time step, we classify a tree as confirmed if at least a branch in a tree has its weight $w_{k}^{i}>w_{T h}$. From each of the confirmed trees, a branch with the highest weight gives the trajectory estimate of a target and is selected into the track set $\Im_{k}$. The label of the selected track is the same as that of the tree it belongs to. We may also pick a branch with the strongest weight at time step $k$ from a tree that was previously confirmed at time step $k-1$ even though the tree has no branch with $w_{k}^{i}>w_{T h}$ at the current time step. This will enable the GM-PHD filter to track target accurately in the presence of target detection uncertainty. A track that was in $\Im_{k-1}$ and not in the current time step $k$, can be regarded as being no longer live in the surveillance region. 


\section{Pruning Schemes}

Tree based structures for managing tags and tracks leads to a number of pruning schemes that are effective and easier to use. Pruning schemes reduce the computational load of the recursion by eliminating the Gaussian components and tracks (represented by the tree branches) that are least likely to represent targets. The likelihoods of Gaussian terms and tracks are determined by their associated weights. It should be noted that these pruning techniques are made possible by the introduction of tags in the GM-PHD filter.

In addition to pruning Gaussian terms of smaller weights according to the pruning step outlined in Section III), we also propose to prune least likely Gaussian terms on the basis of missed detections. We keep a counter $n_{\text {missed }}$ on each branch of a tree to denote the number of consecutive missed detections on that branch. For example, $n_{\text {missed }}$ for the branch represented by branch $h_{1}$ in Figure 2 is two. All branches with an appropriately chosen value of $n_{\text {missed }}$ (three or more) are removed.

We also propose to implement a pruning scheme similar to the $N$-scan pruning used in MHT [27]. Once a track

associated with a Gaussian with $w_{k}^{j}>w_{T h}$ from a tree is chosen as output track, we eliminate all other branches that shares the same node as the chosen branch (or track) at $N$ time steps back.

For all tentative trees, if weights of none of the Gaussians in it reaches $w_{T h}$ in a carefully chosen number of time frames (for example five or more), we delete them.

\section{Simulation Results}

For illustration purposes, we consider a two-dimensional scenario with an unknown and time varying number of targets observed in clutter over the surveillance region $[-500,500] \times[-500,500]$ (in $m$ ). The state $x_{k}=$ $\left[p_{x, k}, \dot{p}_{x, k}, p_{y, k}, \dot{p}_{y, k}\right]^{T}$ of each target consists of its position $\left(p_{x, k}, p_{y, k}\right)$ and velocity $\left(\dot{p}_{x, k}, \dot{p}_{y, k}\right)$, while the measurement is a noisy version of its position. $[\cdot]^{T}$ denotes a transpose of a matrix $[\cdot]$.

Each target follows the linear Gaussian dynamics

$$
x_{k}=\left[\begin{array}{cccc}
1 & T & 0 & 0 \\
0 & 1 & 0 & 0 \\
0 & 0 & 1 & T \\
0 & 0 & 0 & 1
\end{array}\right] x_{k-1}+\left[\begin{array}{cc}
T^{2} / 2 & 0 \\
T & 0 \\
0 & T^{2} / 2 \\
0 & T
\end{array}\right] \sigma_{v}^{2}
$$

with $p_{S, k}=0.90$ and $\sigma_{v}=1$. Each target, if detected, generates an observation according to

$$
z_{k}=\left[\begin{array}{llll}
1 & 0 & 0 & 0 \\
0 & 0 & 1 & 0
\end{array}\right] x_{k}+\sigma_{\epsilon}^{2},
$$

with the detection probability $p_{D, k}=0.98$. The sampling period $T=1$ unit in time and $\sigma_{\epsilon}=\operatorname{diag}([5,5])$.

We assume no spawning, and the spontaneous birth intensity is Poisson with four Gaussian terms distributed across the surveillance region,

$$
\gamma_{k}(x)=\sum_{i=1}^{4} 0.05 \mathcal{N}\left(x ; m_{\gamma, i}, P_{\gamma}\right)
$$


giving the mean number of spontaneously appearing targets at any time, $\lambda_{b}$ is 0.2 . We use $P_{\gamma}=$ $\operatorname{diag}([176400,25,17600,25])$ and targets are born with mean positions of $[250,0,250,0]$ and $[-250,0,-250,0]$.

The detected measurements of targets are immersed in clutter that is typically modelled with as a Poisson RFS with intensity function

$$
\kappa_{k}(z)=\lambda_{c} V u(z)
$$

where $u(\cdot)$ represents the uniform density over the surveillance region, and $\lambda_{c}=4 \times 10^{-5} m^{-2}$ is the average number of clutter returns per unit hyper volume. This translates to 10 clutter measurements per scan.
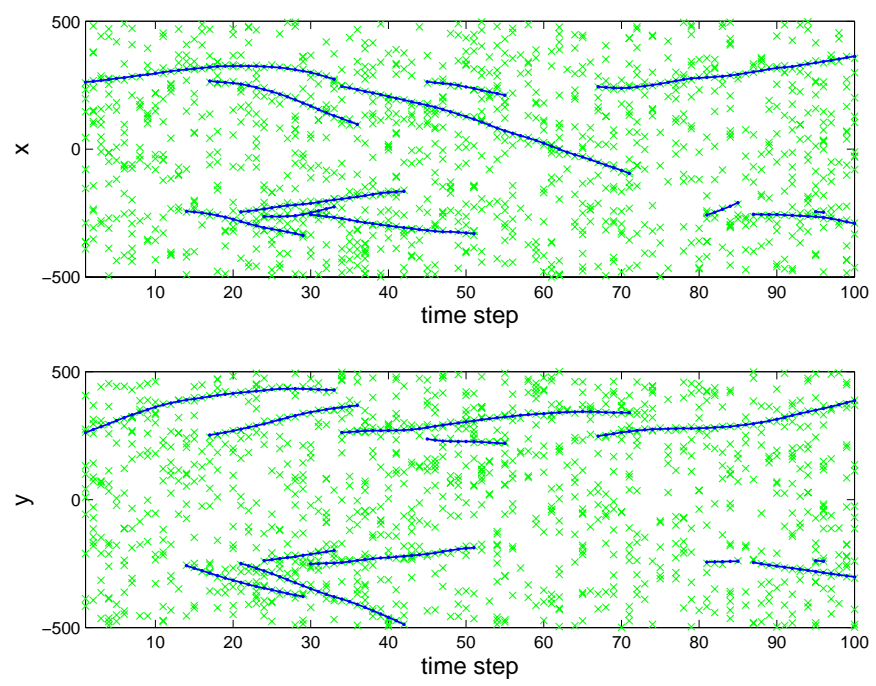

Fig. 3. True target positions (star) superimposed on the measurements generated (cross).

In the GM-PHD filter, pruning parameter threshold $T_{T h}=10^{-5}$, merging threshold $U=4$, weight threshold $w_{T h}=0.5$ and maximum number of Gaussian terms $J_{\max }=200$. We implemented a track-oriented MHT [27] with a batch of 10 frames, in which the log-likelihood ratio was used to rank tracks and the best global hypothesis was considered for data outputs. It should be noted that the MHT implemented for comparison is an approximation, not the full MHT and is for the purpose of benchmarking the GM-PHD tracker.

Figure 3 shows a simulated scenario with true target trajectories together with measurements generated at the sensor for duration of 100 time steps in the presence of clutter. Figure 4 gives the results of the GM-PHD tracker and shows that the GM-PHD tracker gives good estimates of true target trajectories. The estimates given by the GM-PHD tracker is almost free of false tracks. Figure 5 shows estimates of the target trajectories given by a trackoriented MHT that has been used here to benchmark the performance of the GM-PHD tracker. In comparison, the MHT picks up more false tracks as well as occasionally failing to pick up some true tracks. The ability to minimize the number of false tracks picked up by MHT depends on the particular MHT implementation. By choosing a larger number of target detection-hits for a track to be confirmed during track confirmation will reduce the number of false tracks albeit causing true tracks to be lost more often. Since only the confirmed tracks are considered for output in the MHT, any track that does not exist in the surveillance region for long enough will not be picked up 
by MHT.

The performance of the GM-PHD tracker is also benchmarked against that of the MHT in terms the multi-target miss distance and the error in estimation of target number. Five hundred sets of measurements for these target trajectories are been generated and average of these simulation runs are presented in the rest of this section.
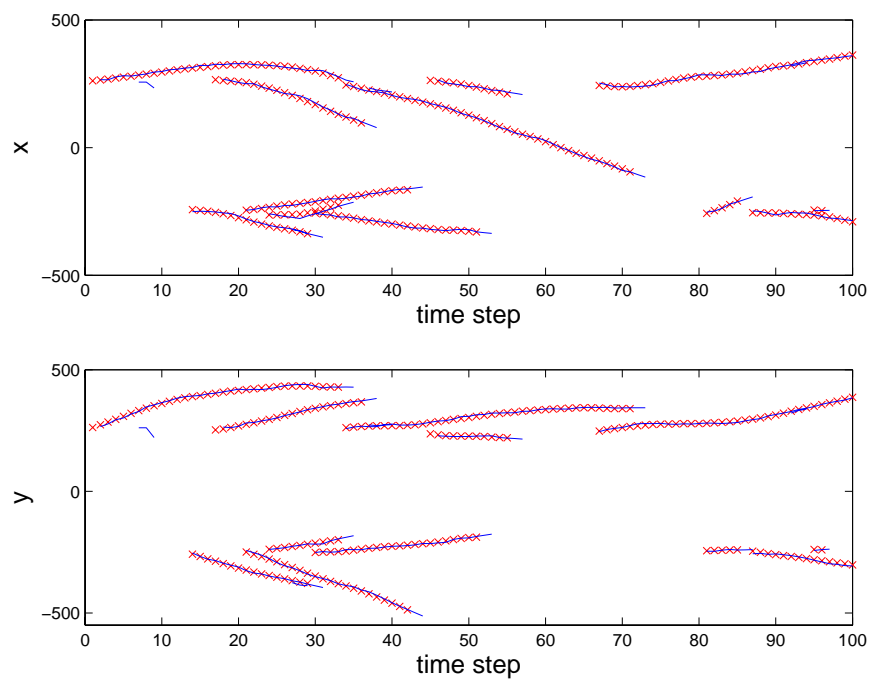

Fig. 4. Target tracks obtained using GM-PHD tracker (solid lines) superimposed with the true target positions (crosses).
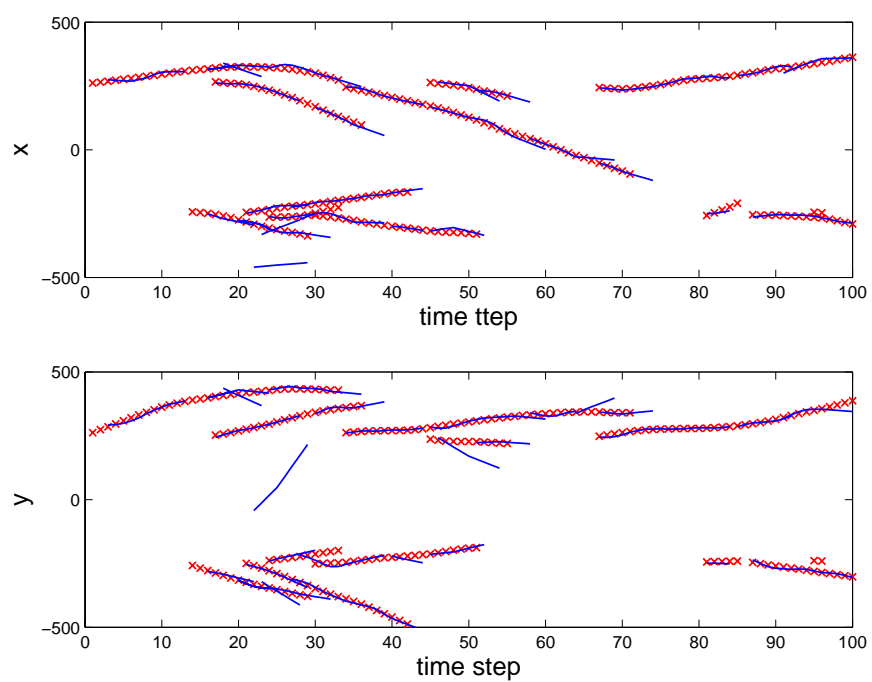

Fig. 5. Target tracks obtained using a track-oriented MHT (solid lines) superimposed with the true target positions (crosses).

\section{A. Wasserstein distance}

The Wasserstein distance from theoretical statistics was adopted as a means of defining a metric for multi-target distances since it penalizes when its estimate of the number of targets is incorrect [28]. This metric has been used for assessing the performance of the PHD filter [12], [19]. 
TABLE I

AVERAGE OF WASSERSTEIN DISTANCE FOR DIFFERENT $\lambda_{c}$ PER $4 \times 10^{-6} m^{-2}$

\begin{tabular}{|c|c|c|c|c|}
\hline & $\lambda_{c}=5$ & $\lambda_{c}=10$ & $\lambda_{c}=15$ & $\lambda_{c}=20$ \\
\hline GM-PHD Tracker & 6.2530 & 40.1625 & 50.9499 & 56.8149 \\
\hline MHT & 130.5379 & 150.7159 & 193.9543 & 224.0479 \\
\hline
\end{tabular}

Given the multi-target ground truth $X=\left\{x_{1}, \cdots, x_{|X|}\right\}$ and its estimate $\widetilde{X}=\left\{\widetilde{x}_{1}, \cdots, \widetilde{x}_{|\widetilde{X}|}\right\}$, the $L^{p}$ Wasserstein distance $d_{p}^{W}$ is defined as follows:

$$
d_{p}^{W}(\tilde{X}, X)=\min _{C} \sqrt[p]{\sum_{i=1}^{|\widetilde{X}|} \sum_{j=1}^{|X|} C_{i, j}\left\|\widetilde{x}_{i}-x_{j}\right\|^{p}},
$$

where the minimum is taken over the set of all transportation matrices $C=\left\{C_{i, j}\right\}$; and each entry of the matrix $C$ satisfies the followings: $C_{i, j} \geq 0, \sum_{i=1}^{|\tilde{X}|} C_{i, j}=1 /|\tilde{X}|$ and $\sum_{j=1}^{|X|} C_{i, j}=1 /|X|$. In this work, $p$ takes a value of two.

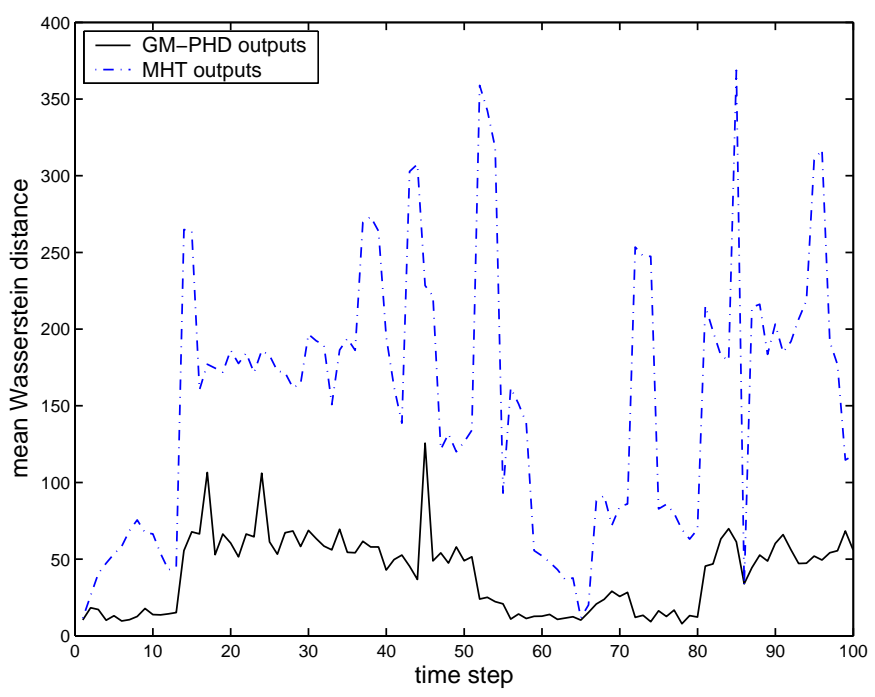

Fig. 6. Error in the number of target estimates for the GM-PHD tracker and a track-oriented MHT.

Figure 6 shows the Wasserstein distance averaged over for 500 measurement sets for 100 time steps. The occasional spikes in the plot of the Wasserstein distance for the GM-PHD tracker is usually due to the fact that either a new target has entered the surveillance region and has not yet been detected, or a target has just died and has not yet been eliminated. In comparison, the plot of Wasserstein distance for the MHT has larger peaks more frequently. Moreover, the Wasserstein distance for the GM-PHD tracker is consistently smaller than that for the MHT as GM-PHD tracker is more accurate in estimating the number of targets. Table I shows the Wasserstein distance for different clutter ratios and shows that the Wasserstein distance for the GM-PHD tracker is consistently smaller than that of the MHT. 


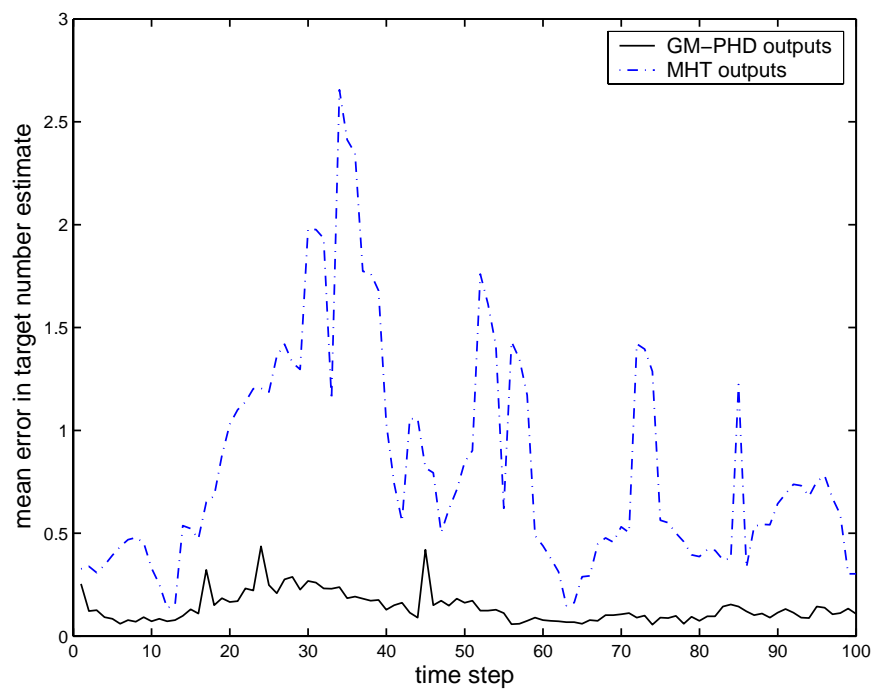

Fig. 7. Mean error in the target number estimates for the GM-PHD tracker and a track-oriented MHT.

TABLE II

ERror In TARget Number Estimate For DifFERENT $\lambda_{c}$ PER $4 \times 10^{-6} \mathrm{~m}^{-2}$

\begin{tabular}{|c|c|c|c|c|}
\hline & $\lambda_{c}=5$ & $\lambda_{c}=10$ & $\lambda_{c}=15$ & $\lambda_{c}=20$ \\
\hline GM-PHD Tracker & 0.0967 & 0.1378 & 0.1769 & 0.2013 \\
\hline MHT & 0.6351 & 0.8261 & 1.1786 & 1.7486 \\
\hline
\end{tabular}

\section{B. Error in Estimating the Number of Targets}

Figure 7 shows the absolute error in estimating the number of targets (i.e., $E\left\{|| X_{k}|-| \hat{X}_{k}||\right\}$ ), averaged over results obtained from 500 measurement sets. (Note that standard performance measures such as the mean square distance error is not applicable to multi-target filters that jointly estimate number of targets and their states.) It shows that the plot of error in estimating target number for the MHT has peaks higher and more often than that for the GM-PHD tracker. The absolute error in total target number estimates is consistently smaller for the GM-PHD tracker than for the MHT. The absolute error in estimating target numbers averaged over 100 time steps is 0.1378 for the GM-PHD tracker and 0.8261 for the MHT. This shows that the GM-PHD tracker provides target number estimates more accurately than the MHT. This observation can also be made from the Table II that shows error in the target number for these two tracker for different clutter rates.

\section{Remarks}

For the linear Gaussian multi-target models, the proposed tracker is an alternative method to the PHD-withassociation filter that is proposed to provide data association for the SMC-PHD filter [19]. In the case of the PHDwith-association filter, clustering techniques that are needed for the state extraction are computationally demanding and might add errors in the target states. 
Data association techniques that are proposed for the SMC-PHD filter [19], [20], [23] can also be applied to the GM-PHD tracker. However, such schemes would require additional steps like prediction and gating, and the required computational cost would be considerably more than the cost of using tags. However, such techniques could be employed in conjunction with the GM-PHD tracker in some situations and will be presented in the following section.

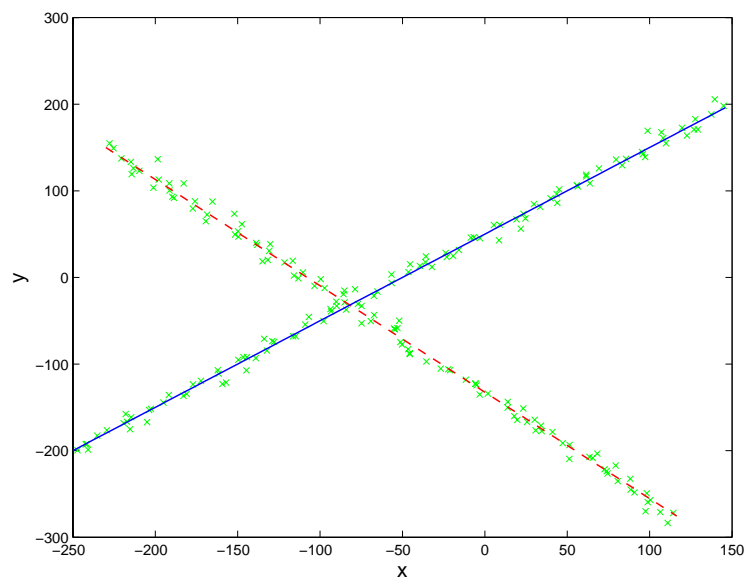

Fig. 8. Crossing target trajectories with target ' 1 ' (dashed room) and 'target 2' (solid line).

\section{THE GM-PHD TRACKER AND CROSSING TARgETS}

The algorithm presented in Section III has a theoretical limitation in that it is unable to distinguish between any two targets when they are within a certain distance of each other [9]. Consider a situation where two targets are in the surveillance region. Ideally the intensity function would be represented by two Gaussians as

$$
v_{k}(x)=w_{k}^{1} \mathcal{N}\left(x ; m_{k}^{1}, P_{k}\right)+w_{k}^{2} \mathcal{N}\left(x ; m_{k}^{2}, P_{k}\right)
$$

(For simplicity, we take the covariance matrix for both Gaussians to be the same.)

Suppose that these two targets cross, then the intensity function $v_{k}$ at time step $k$ is unimodal with the mean $\left(m_{k}^{1}+m_{k}^{2}\right) / 2$ when $\left|m_{k}^{1}-m_{k}^{2}\right|<2\left|P_{k}\right|^{1 / 2}$ [9]. This means that the GM-PHD tracker fails to distinguish between targets within this separation. Thus, if separate tracks of targets are to be maintained when targets are too close, alternative methods for data association are needed. Next, we show that the correct target identities can be maintained by using their past trajectories. Assuming that the targets have been detected and their trajectories up to time step $k-1$ are available, we propose to apply an 'estimate-to-track' association scheme similar to the one proposed in [20] for the SMC-PHD filter.

\section{A. Estimate-to-Track Association for the GM-PHD Tracker}

Given the set of target state estimates $\widehat{X}_{k}$ and their respective tags $\hat{T}_{k}$, we first propose to construct $\widehat{X}_{k}^{1}$ and $\hat{T}_{k}^{1}$ where for every $m_{k}^{i} \in \widehat{X}_{k}^{1}$ there exists at least one other $m_{k}^{j}(i \neq j)$ for which $\left|m_{k}^{i}-m_{k}^{j}\right|<2\left|P_{k}\right|^{1 / 2}$ and $\hat{T}_{k}^{1}$ denotes 


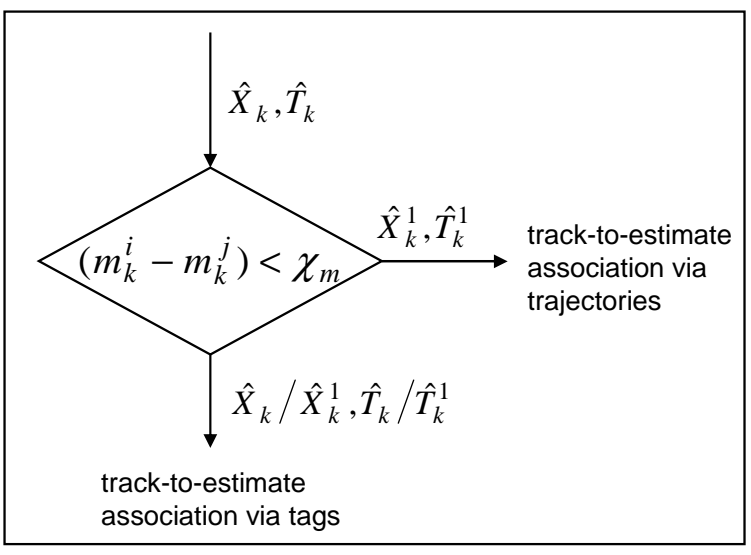

Fig. 9. An schematic view of extending the GM-PHD filter to crossing targets.

the set of tags of each $m_{k}^{j} \in \widehat{X}_{k}^{1}$. Essentially, $\widehat{X}_{k}^{1}$ contains all multiple Gaussian terms that are within merging threshold. Given the track set $\Im_{k-1}$ at time step $k-1$, the estimate-to-track association for the state estimates in $\widehat{X}_{k} \backslash \widehat{X}_{k}^{1}$ are performed according to the scheme presented in Section III.

To resolve the track identities of target state estimates present in $\widehat{X}_{k}^{1}$, the trajectories of these targets upto time step $k-1$ are obtained from the track set $\Im_{k-1}$ and initialized as track hypotheses. The identity of a track hypothesis is given by the label of the initializing track. For each track hypothesis, we propagate its mean and covariance as $\tilde{m}_{k \mid k-1}^{i}=F_{k-1} \tilde{m}_{k-1}^{i}$, and $\tilde{P}_{k \mid k-1}^{i}=Q_{k-1}+F_{k-1} P_{k-1}^{j}\left(F_{k-1}\right)^{T}$. The score of the track is represented by its $\log$-likelihood ratio (LLR) that is initialized with $\log \left(w_{k-1}^{i}\right)$. For each track, an association track hypothesis is formed by associating it with every $m_{k}^{j} \in \widehat{X}_{k}^{1}$, and its $L L R$ is incremented as

$$
L L R_{k, i}^{j}=L L R_{k-1, i}+\log \left(\mathcal{N}\left(m_{k}^{j} ; \tilde{m}_{k \mid k-1}^{i}, P_{k}^{j}+\tilde{P}_{k \mid k-1}^{i}\right)\right)
$$

Figure 10 shows an example of two crossing targets with $\left|m_{k}^{i}-m_{k}^{j}\right|<2\left|P_{k}\right|^{1 / 2}$. Trees are initialized with the previous trajectories and labels at time step $k-1$, and association hypotheses are formed with both means $m_{k}^{i}$ and $m_{k}^{j}$ at time steps $k$ and $k+1$. The objective here is to resolve the labels of $m_{k}^{i}$ and $m_{k}^{j}$ at time step $k$ and onwards.

At time step $k$, we have a number of hypothesis trees and a branch of a tree is an association track hypothesis and represents the same target. At time step $k$, a branch of the highest $L L R$ from each hypothesis tree denotes a possible track. The identity of the track and that of every Gaussian that belongs to it is given by the tree it belongs to. We propose to recursively extend each tree by forming association hypotheses for every branch with $\widehat{X}_{k}^{1}$ that arrives next in time. Once $\left|m_{k}^{i}-m_{k}^{j}\right|$ is no longer smaller than $2\left|P_{k}\right|^{1 / 2}$ for any $m_{k}^{i}$ and $m_{k}^{j}$ in $\widehat{X}_{k}^{1}$, an association track hypothesis of the the highest LLR is selected from each tree and are passed back to the GM-PHD tracker along their labels.

\section{B. Example of Crossing Targets}

For illustration purposes, Figure 8 shows two targets in the surveillance region whose trajectories cross each other at time step $k=53 \mathrm{~s}$. Figures 11 and 12 show the results of two different simulation runs for the targets given 


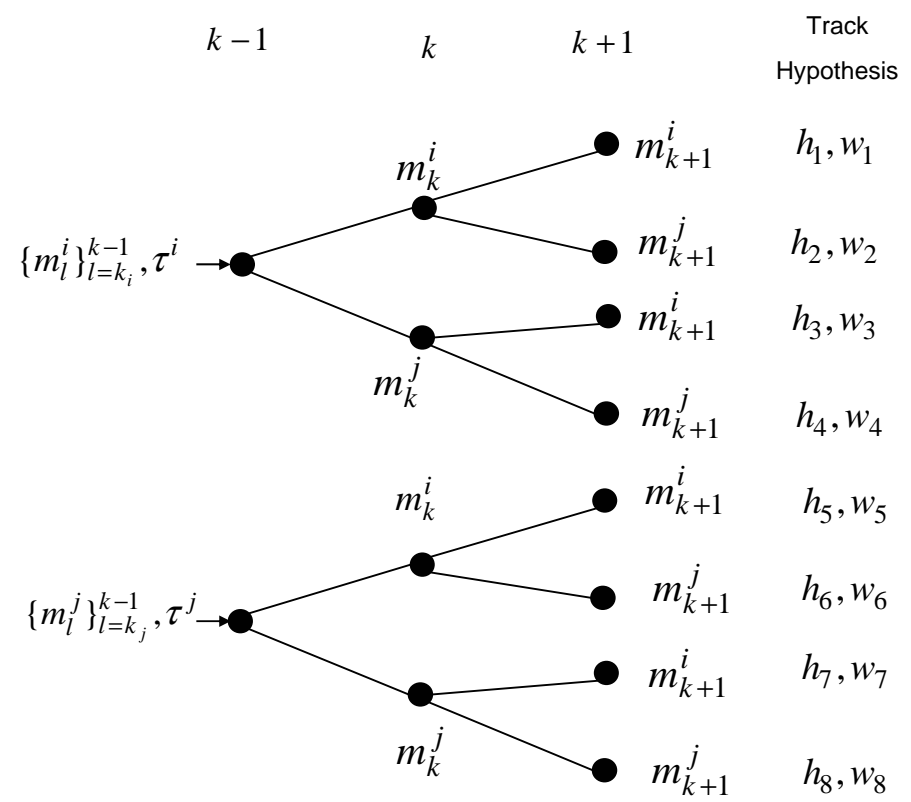

Fig. 10. An example of estimate-to-track association for the crossing targets in the GM-PHD filter. $w_{i}$ denotes the log-likelihood ratio (LLR) of a track hypothesis at the current time step, i.e. $k=2$ in this case.

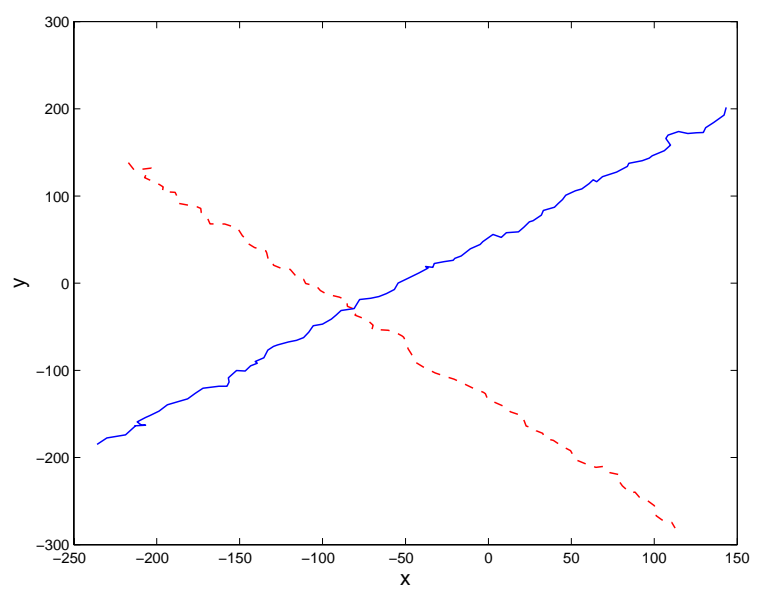

Fig. 11. Trajectories (identified correctly) given by the GM-PHD tracker for crossing targets.

in Figure 8. While results presented in Figure 11 correctly keep the target identities of two targets, the GM-PHD tracker fails to correctly keep separate target identities as shown in Figure 12. Another possible outcome would be the assignment of the same identity to both of these tracks.

Figure 13 shows the results of the improved GM-PHD tracker that consistently resolves the track identities of the crossing targets. However this will require the computation of distances amongst all target estimates at every time steps and as a result adds to the computational load of the overall GM-PHD tracker.

\section{CONCLUSION}

This paper presents a novel GM-PHD filter based multi-target tracker that can correctly track multiple targets in noisy sets of measurements in the presence of ambiguous origin and missed detections. The GM-PHD tracker assigns tags to individual Gaussian terms and uses tree structures for propagating these tags that provide track 


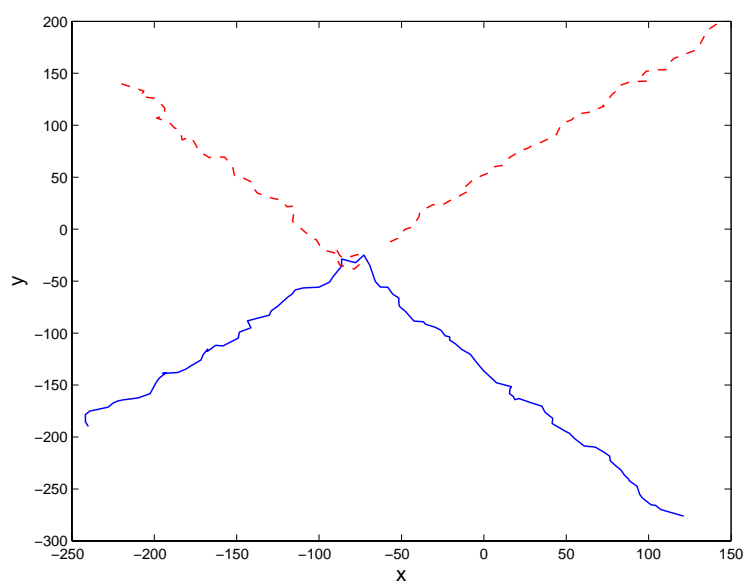

Fig. 12. Trajectories (wrongly identified) given by the GM-PHD tracker for crossing targets.

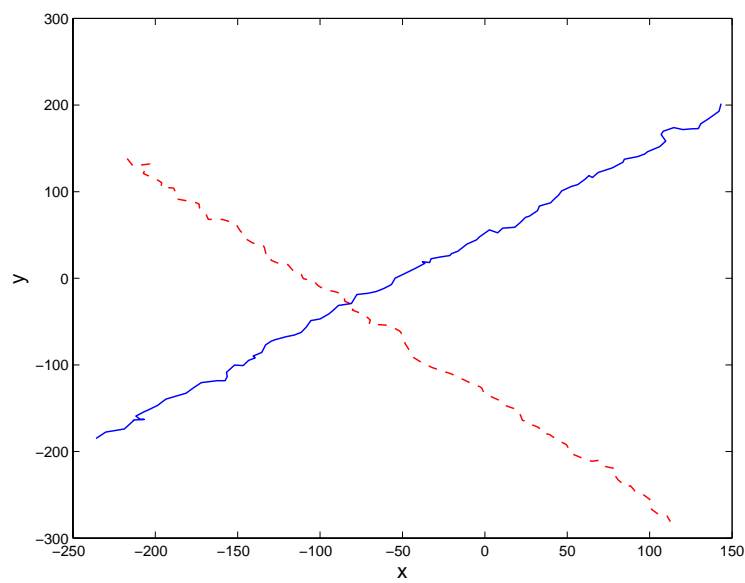

Fig. 13. Trajectories given by the GM-PHD tracker that performs track-to-estimate association between time steps 52 and 55 .

labels. An efficient track management scheme has been proposed for the tracker to allow track initiation, track propagation and track termination. The proposed methods and their processing are performed in parallel and do not affect the GM-PHD recursion. The computational load of initializing the track and performing the track-to-estimate association is minimal compared with the recursion of the Gaussian mixture components approximating the intensity function. This paper also includes a number of methods to prune Gaussian terms that are least likely represents targets. The performance of the GM-PHD tracker is benchmarked against that of the MHT for a multi-target tracking example. It is shown that the GM-PHD tracker operates with a fewer number of false tracks and smaller multi-target miss distance. However, we must bear in mind that the performance MHT schemes depend on their particular implementations and how much computational load they are prepared to tolerate. This paper also includes a discussion on the theoretical constraints of the proposed tracker in its ability to maintain separate track identities of targets that are crossing or are in close proximity. We have presented a technique for extending the ability of the GM-PHD tracker to handle crossing targets. However, further study is needed to study the computational burden of extending the GM-PHD tracker to crossing targets as we need to calculate distances amongst target state estimates 
to determine which target estimates are to be subjected to track-to-estimate association.

\section{ACKNOWLEDGEMENTS}

This work was supported by Co-operative Research Centre for Sensor Signal and Information Processing (CSSIP), Department of Electrical \& Electronic Engineering, The University of Melbourne, Parkville, Victoria 3010, Australia.

\section{REFERENCES}

[1] Y. Bar-Shalom and T. E. Fortmann, Tracking and Data Association, Academic Press, Boston, 1988.

[2] Y. Bar-Shalom and X. R. Li, Multitarget-Multisensor Tracking: Principles and Techniques, Storrs, CT: YBS Publishing, 1995.

[3] S. Blackman and R. Popoli, Design and Analysis of Modern Tracking Systems, Artech House, Norwood, 1999.

[4] D. Daley and D. Vere-Jones, An Introduction to the Theory of Point Processes. Springer-Verlag, 1988.

[5] D. Stoyan, D. Kendell and J. Mecke, Stochastic Geometry and its Applications. John Wiley \& Sons, 1995.

[6] I. Goodman, R. Mahler and H. Nguyen, Mathematics of Data Fusion, Kluwer Academic Publishers, Dordrecht/Boston/London, 1997.

[7] R. Mahler, An Introduction to Multisource-Multitarget Statistics and its Applications, Lockheed Martin Technical Monograph, March $15,2000$.

[8] R. Mahler, "Random set theory for target tracking and identification," Data Fusion Handbook, D. Hall and J. Llinas (eds.), CRC press Boca Raton, pp. 14/1-14/33, 2001.

[9] R. Mahler, "Multi-target Bayes filtering via first-order multi-target moments," IEEE Trans. on Aerospace and Electronic Systems, Vol. 39, No. 4, pp. 1152-1178, Oct. 2003.

[10] M. Vihola, Random Sets for Multitarget Tracking and Data Fusion, Licentiate Thesis, Tampere University of Technology, 2004.

[11] B. Vo, S. Singh and A. Doucet, “Sequential Monte Carlo Implementation of the PHD Filter for Multi-Target Tracking," in Proc. of Fusion, 2003, pp. 792-799, Cairns, Australia.

[12] B. Vo, S. Singh and A. Doucet, "Sequential Monte Carlo Methods for Multi-target Filtering with Random Finite Sets," IEEE Trans. Aerospace and Electronic Systems, Volume 41, Issue 4, pp.1224-1245, Oct., 2005.

[13] B. Vo and W. K. Ma, "A Closed Form Solution for The Probability Hypothesis Density Filter" Proc. 7th International Conference on Information Fusion, 2005, Vol. 2, 25-28 July 2005. pp. 856-863.

[14] B. Vo and W. K. Ma, "The Gaussian Mixture Probability Hypothesis Density filter", IEEE Trans. Signal Processing, Vol. 54, No. 11, pp. 4091-4104, 2006.

[15] W. K. Ma , B. Vo, S. Singh and A. Baddeley, 'Tracking an unknown time-varying number of speakers using TDOA measurements: A Random Finite Set Approach ”, IEEE Trans Signal Processing, Vol. 54, No. 9, pp. 3291-3304, 2006.

[16] H. Sidenbladh and S. Wirkander, "Tracking Random Sets of Vehicles in Terrain," Proc. of IEEE Workshop on Multi-Object Tracking, Madisson, Wisconsin, 2003.

[17] H. Sidenbladh, "Multi-Target Particle Filtering for the Probability Hypothesis Density,” Proc. of Fusion'2003, pp. 1110-1117, Cairns, Australia.

[18] T. Zajic, and R. Mahler, "A Particle-Systems implementation of the PHD multi-target tracking filter," in I. Kadar (ed.), Signal Processing, Sensor Fusion and Target Recognition XII, Proc. SPIE, Vol. 5096, pp. 291-299, 2003.

[19] K. Panta, B. Vo, S. Singh and A. Doucet, "Probability Hypothesis Density Filter versus Multiple Hypothesis Tracking," Proc. of SPIE, Signal Processing, Sensor Fusion \& Target Recognition XIII, Vol. 5429, I.d Kader, Editor, August 2004, pp. $284-295$.

[20] K. Panta, B. Vo and S. Singh, "Novel Data Association Techniques for the Probability Hypothesis Density Filter," to appear in IEEE Trans. on Aerospace and Electronic Systems, Vol. 43, No. 2, July, 2007.

[21] L. Lin, Y. Bar-Shalom and T. Kirubarajan, “Track Labelling and the PHD Filter for Multitarget Tracking," IEEE Trans. on Aerospace and Electronic System, Vol. 42(3), pp. 778-795, 2006. 
[22] K. Panta, B. Vo and S. Singh, “The Improved Probability Hypothesis Density (PHD) Filter for Multitarget Tracking," Proc. Third International Conference on Intelligent Sensing and Information Processing, 14-17 December, 2005. pp. 213-218.

[23] D. E. Clark and J. Bell, "Data Association for the PHD Filter," Proc. Second International Conference on Intelligent Sensor, Sensor Networks and Information Processing, 5-8 December, 2005. pp. 217-222, Melbourne, Australia.

[24] D. E. Clark, K. Panta and B. Vo, "GM-PHD Filter Multi-target Tracker", In Proceedings of 9th International Conference on Information Fusion, 10-13 July 2006. Florence, Italy.

[25] D. E. Clark and B. Vo, "Convergence Analysis of the Gaussian Mixture PHD Filter" IEEE Transactions on Signal Processing, Vol. 55, Issue 4, pp. 1204-1212, April 2007.

[26] K. Panta, B. Vo and D. E. Clark, “An Efficient Track Management Scheme for the Gaussian-Mixture Probability Hypothesis Density (GM-PHD) Tracker," to appear in Proc. Fourth International Conference on Intelligent Sensing and Information Processing, 15-18 December, 2006.

[27] T. Kurien, "Issues in the design of practical multitarget tracking algorithms," in Multitarget-Multisensor Tracking: Adavanced Applications, Y. Bar-Shalom, ed., pp. 43-83, Artech House, Norwood, 1990.

[28] J. Hoffman and R. Mahler, "Multi-Target Miss Distance via Optimal Assignment," IEEE Trans. Sys., Man., and Cybernetics-Part A, Vol. 34, No. 3, pp. 327-336, May, 2004.

\section{Table III: Pseudo-code for the Gaussian Mixture PHD Tracker}

step 0. (Initialization at $k=0$.)

Initialize the algorithm with the weighted sum of $J_{0}$ Gaussians,

$$
v_{0 \mid 0}=\sum_{i=1}^{J_{0}} w_{0}^{i} \mathcal{N}\left(x ; m_{0}^{i}, P_{0}^{i}\right),
$$

assign a tag to each Gaussian component,

$$
\mathcal{T}_{0}:=\left\{\tau_{0}^{i}, \ldots, \tau_{0}^{J_{0}}\right\}
$$

initialize the track set with an empty set

$$
\Im_{0}:=\emptyset
$$

set $k=1$.

step 1. (Prediction Step, for $k \geq 1$.)

Predict existing Gaussian components with Kalman filter,

$$
v_{S, k \mid k-1}(x)=p_{S, k} \sum_{j=1}^{J_{k-1}} w_{k-1}^{j} \mathcal{N}\left(x ; m_{S, k \mid k-1}^{j}, P_{S, k \mid k-1}^{j}\right),
$$

Introduce $J_{\gamma, k}$ components for the spontaneous birth model,

$$
\gamma_{k}(x)=\sum_{i=1}^{J_{\gamma, k}} w_{\gamma, k}^{i} \mathcal{N}\left(x ; m_{\gamma, k}^{i}, P_{\gamma, k}^{i}\right),
$$

The intensity, $v_{k \mid k-1}$, to time step $k$ is then

$$
v_{k \mid k-1}(x)=v_{S, k \mid k-1}(x)+v_{\beta, k \mid k-1}(x)+\gamma_{k}(x),
$$

Concatenate spontaneous birth tag with prediction tags,

$$
\mathcal{T}_{k \mid k-1}=\mathcal{T}_{k} \cup\left\{\tau_{\gamma_{k}}^{1}, \ldots, \tau_{\gamma_{k}}^{J_{\gamma_{k}}}\right\} \cup\left\{\tau_{\beta_{k}}^{1}, \ldots, \tau_{\beta_{k}}^{J_{\beta_{k}}}\right\} .
$$

step 2. (Update Step, for $k \geq 1$.)

When measurements, $Z_{k}=\left\{z_{k, 1}, \ldots, z_{k,\left|Z_{k}\right|}\right\}$, received at time step $k$, update intensity with Kalman filter,

$$
v_{k \mid k}(x)=\left(1-p_{D, k}\right) v_{k \mid k-1}(x)+\sum_{z \in Z_{k}} \sum_{j=1}^{J_{k \mid k-1}} w_{k}^{j}(z) \mathcal{N}\left(x ; m_{k \mid k}^{j}(z), P_{k \mid k}^{j}\right)
$$




$$
w_{k}^{(j)}(z)=\frac{p_{D, k} w_{k \mid k-1}^{j} q_{k}^{j}}{\lambda_{c} c_{k}(z)+p_{D, k} \sum_{\ell=1}^{J_{k \mid k-1}} w_{k \mid k-1}^{\ell} q_{k}^{l}} .
$$

For each prediction component, assign the label to all the updated Gaussian terms.

step 3. (State Estimation, for $k \geq 1$.)

Target states are determined from Gaussians whose weights are above a specific threshold.

$$
\hat{T}_{k}=\left\{\tau_{k}^{i}: w_{k}^{i}>w_{t h}\right\}
$$

The set of estimates is

$$
\hat{X}_{k}=\left\{m_{k}^{i}: \tau_{k}^{i} \in \hat{T}_{k}, i=1, \cdots J_{k}\right\} .
$$

step 4. (Track-to-estimate Association for $k \geq 1$.)

For each $\tau_{k}^{i} \in \hat{T}_{k}$, if there exists a track in $\Im_{k-1}$ with identity $r=\tau_{k}^{i}$, append $m_{k}^{i}$ to the track with identity $r=\tau_{k}^{i}$ and copy it into $\Im_{k}$, missed-estimate counter $=0$,

else

find $m_{l}^{i}$ with tag $\tau_{k}^{i}$ for $l=k-K: k$ and initialize a track with $\left\{m_{k-K: k}^{i}\right\}$ with track identity $r=\tau_{k}^{i}$ in $\Im_{k}$, missed-estimate counter $=0$,

end

For each track $\Im_{k-1}$ that is not copied into $\Im_{k}$, if missed-estimate counter $=0$, append the track with $m_{k}^{r}=F_{k} m_{k \mid k-1}^{r}$ and copy the track into $\Im_{k}$, set missed-estimate counter $=0$.

end.

step 5. (Output)

Target state estimates: $\hat{X}_{k}=\left\{m_{k}^{i}: \tau_{k}^{i} \in \hat{T}_{k}, j=1, \ldots k\right\}$,

Track valued estimates: $\Im_{k}$.

Kusha Panta Biography text here.

Daniel E. Clark Biography text here. 